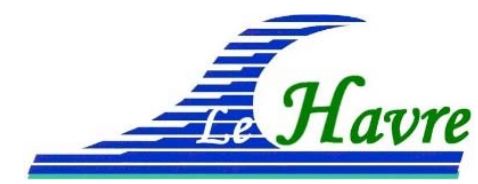

XVI İmes Journées Nationales Génie Côtier - Génie Civil

Le Havre, 2020

DOI:10.5150/jngcgc.2020.041 C Editions Paralia CFL

disponible en ligne - http://www.paralia.fr - available online

\title{
Détection du colmatage des milieux à double porosité par ondes ultrasonores
}

\author{
Fatima Zahraa KACHKOUCH ${ }^{1}$, Abdellah ALEM ${ }^{1}$, Alain TINEL ${ }^{1}$, \\ Hervé FRANKLIN ${ }^{1}$, Huaqing WANG ${ }^{1}$
}

\author{
1. Laboratoire Ondes et Milieux Complexes UMR CNRS 6294, Université Le Havre \\ Normandie, 75, rue Bellot 76600 Le Havre France. \\ fatima-zahraa.kachkouch@univ-lehavre.fr
}

\section{Résumé :}

Le phénomène du colmatage affectant les sites géologiques constitués de matériaux à porosité multiple est dû au dépôt de particules fines dans les pores du matériau. Il est difficilement détectable lors de ses premières manifestations. L'objectif est de proposer une technique non destructive basée sur des mesures ultrasonores, pour la détection du colmatage. Cette technique est confrontée à la méthode destructive couramment employée dans les études en laboratoire, pour les problèmes de transfert en milieux poreux : le matériau est détruit afin d'évaluer par pesées le dépôt, dans différentes couches du milieu. Un modèle expérimental combinant les deux techniques est développé à cet effet. Nous avons examiné deux matériaux granulaires pouvant être assimilés à des milieux à double porosité mais de comportements différents ainsi qu'un matériau à simple porosité.

Le suivi du colmatage est réalisé dans l'espace et dans le temps. L'évolution des propriétés acoustiques - en l'occurrence le coefficient de transmission - est présentée avant et après injection de particules, et permet de tracer le profil de la variation de l'énergie $\Delta \mathrm{E}$ du signal transmis à travers le milieu. Cette variation est en relation directe avec le profil de dépôt. Les résultats obtenus montrent un bon accord entre les deux méthodes utilisées.

\section{Mots-clés :}

Colmatage, Milieux granulaires, Double porosité, Ondes acoustiques, Ultrasons, Dépôt de particules, Transmission.

\section{Introduction}

Lors de l'écoulement d'un fluide chargé de particules fines dans les ouvrages hydrauliques constitués de milieux à porosité simple (SPP) ou double porosité (DPP), plusieurs phénomènes sont susceptibles de se produire et peuvent avoir des conséquences néfastes sur le bon fonctionnement de l'ouvrage : le dépôt de particules peut provoquer un colmatage ; il a été montré par MOGHADASI et al. (2004) que le colmatage des puits de récupération provient de la présence de particules fines dans l'eau. L'une des caractéristiques principales d'un matériau poreux est de permettre une bonne circulation 


\section{Thème 3 - Instrumentation, mesures, imagerie et télédétection}

du fluide notamment l'eau par le biais d'une perméabilité assez suffisante. La chute de perméabilité, qui est l'une des conséquences les plus importantes lorsque le milieu subit un phénomène de colmatage (ALEM et al., 2013), peut nuire au bon fonctionnement de l'ouvrage.

Dans ce travail, nous proposons, de détecter expérimentalement l'accumulation de particules déposées dans les milieux poreux par une méthode ultrasonore non destructive. Ceci nécessite dans un premier temps de comprendre comment les ondes acoustiques se propagent à travers de tels milieux complexes. Le modèle décrivant la propagation des ondes acoustiques dans les milieux à simple porosité saturés est celui de BIOT (1956). Notre choix d'étudier un milieu à double porosité est basé sur le fait que dans la nature, les systèmes aquifères présentent généralement des hétérogénéités granulométriques et/ou structurales. C'est pour cette raison notamment, la théorie de Biot a été étendue (WARREN \& ROOT, 1963 ; WILSON \& AIFANTIS, 1984 ; BERRYMAN \& WANG, 2000) aux milieux à double porosité.

L'analyse du dépôt de particules dans le milieu poreux saturé, lorsque celui-ci est soumis à un écoulement de particules en suspension, est quantifiée par des mesures de la propagation des ondes acoustiques en transmission. Des traçages par injection continue sur une colonne contenant le milieu poreux sont réalisés parallèlement afin d'étudier la rétention des particules. Les résultats de mesure du dépôt et mesures ultrasonores sont confrontés, l'objectif étant de mettre en évidence l'influence du dépôt de particules sur les comportements mécanique et acoustique du milieu.

\section{Description des matériaux utilisés}

Trois matériaux poreux granulaires sont utilisés : deux milieux à double porosité de comportements mécaniques différents, et un milieu à simple porosité servant de référence. Ce dernier - BV - est formé par empilement de billes de verre sphériques de diamètre 3,5 mm. Le premier milieu à double porosité - Robu - est constitué de billes de verres poreuses borosilicate 3.3 (VitraPOR ${ }^{\circledR}$ Sinterfilter \& Laboratory Glassware) (figures 1a, 1b). Il est largement utilisé dans le domaine de la recherche en raison de sa résistance à la chaleur, à la corrosion et aux chocs thermiques. Le deuxième-Tobermorite - est un ensemble de grains poreux de matière semi-naturelle Tobermorite $11 \AA$ (grains de ciment poreux de forme irrégulière, et dimensionnés de 3,15 $\mathrm{mm}$ à $4 \mathrm{~mm}$ obtenus par tamisage) (figures 1c,1d). Robu et Tobermorite présentent une double porosité : une macroporosité entre les grains et une microporosité à l'intérieur des grains (figures $1 \mathrm{~b}$, 1d).

La porosité totale de chacun des trois échantillons mesurée à l'aide du pycnomètre à air D5550-6 est de 39\%, 60\% et 70\% pour BV, Robu et Tobermorite, respectivement. Plusieurs paramètres physiques caractérisent les matériaux décrits ci-dessus; les valeurs numériques sont tirées pour les billes de verre de (KIMURA, 2006), et pour les deux milieux à double porosité de (BAI et al., 2018). 


\section{XVI'̀mes Journées Nationales Génie Côtier - Génie Civil \\ Le Havre 2020}

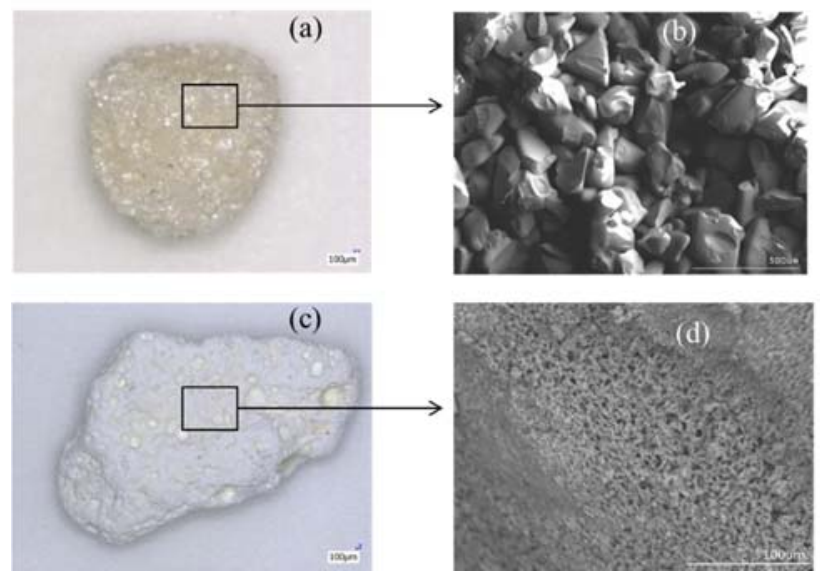

Figure 1. (a) Un grain de Robu (taille de 3,5 mm), (b) vues au MEB, (c) Un grain de Tobermorite 11 A (taille de $2 \mathrm{~mm}$ à $3 \mathrm{~mm}$ ), (d) vues au MEB.

\section{Dispositif expérimental}

Le protocole expérimental se compose d'un couplage de deux types d'équipements (figure 2): le premier permettant d'effectuer des mesures acoustiques, et le second consistant à faire des mesures de la rétention de particules pour évaluer le colmatage. Le matériau poreux (SPP ou DPP) est placé dans une colonne rectangulaire en aluminium de dimensions $100 \times 100 \times 450 \mathrm{~mm}^{3}$ (l'épaisseur de la paroi est de $2 \mathrm{~mm}$ ). La colonne est placée horizontalement afin d'éviter un tassement éventuel provoquant l'hétérogénéité du matériau et est entièrement immergée dans une grande cuve remplie d'eau pendant au moins 24 heures afin que les milieux poreux soient totalement saturés.

Pour effectuer des mesures ultrasonores, deux transducteurs à immersion - $\varnothing 5 \mathrm{~cm}$ et fréquence centrale $44 \mathrm{kHz}$ - positionnés l'un en face de l'autre (sans contact avec les plaques métalliques) sont utilisés. A l'aide d'un système mécanique de translation, ces transducteurs (l'un agissant comme émetteur d'ondes acoustiques, l'autre comme récepteur) peuvent se déplacer le long de l'axe horizontal (figure 2) (huit positions différentes sont définies pour les résultats). Un générateur Sofranel (modèle 5058 PR) délivre des impulsions d'amplitude $300 \mathrm{~V}$ et de durée $200 \mathrm{~ns}$; le signal de synchronisation de l'oscilloscope est également délivré par le générateur. L'impulsion électrique est envoyée au transducteur émetteur, qui la convertit en onde acoustique. Celle-ci, dans le milieu, se convertit en trois ondes (milieu SPP) ou quatre ondes (milieu DPP), qui sont captées et converties de nouveau en un signal électrique par le transducteur récepteur. Les signaux reçus sont numérisés et moyennés sur 200 acquisitions par un oscilloscope numérique de marque Lecroy $9310 \mathrm{AL}$ (400 MHz de bande passante). Ils sont ensuite stockés dans un ordinateur relié à l'oscilloscope. 


\section{Thème 3 - Instrumentation, mesures, imagerie et télédétection}

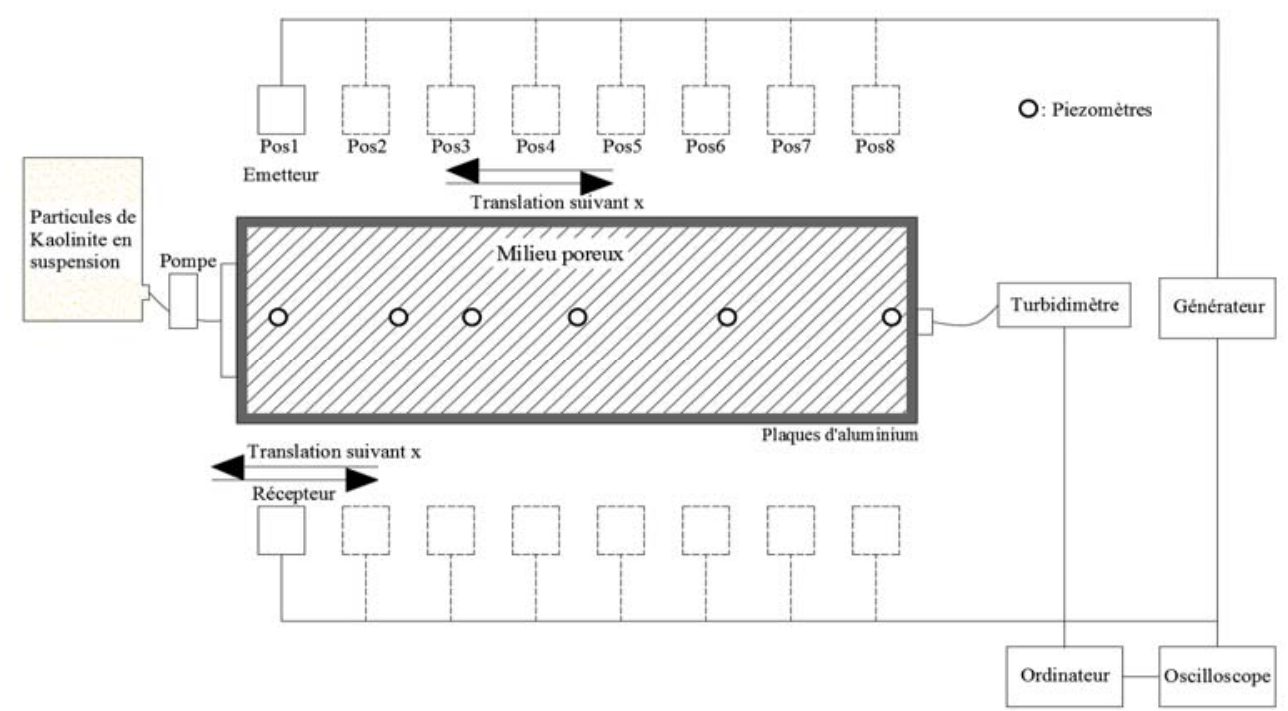

Figure 2. Dispositif expérimental pour les analyses acoustique et mécanique du phénomène de colmatage.

Concernant le second équipement des mesures de dépôt, la colonne est alimentée par des réservoirs contenant de l'eau ( $\mathrm{pH}$ de $6,7 \pm 0,2)$ et des particules en suspension (SusP) (particules de kaolinite de Portland d'un diamètre moyen de $11 \mu \mathrm{m}$ ) à l'aide d'une pompe péristaltique Cole-Parmer Master flex avec contrôle du débit. La suspension est injectée à travers le milieu poreux sur une longue durée à débit constant. Six piézomètres sont placés sur la colonne comme indiqué sur la figure 2 pour mesurer la variation de la pression interstitielle le long du milieu poreux. À la sortie de la colonne, l'effluent traverse un turbidimètre à écoulement continu (Kobold Instruments) pour enregistrer les valeurs de turbidité en termes d'Unités de Turbidité Néphélométrique (NTU). Par la suite, les concentrations de particules en suspension dans l'effluent, notées $\mathrm{C}$, sont déterminées à l'aide de corrélations établies entre les concentrations mesurées de particules en suspension dans l'eau et les valeurs en NTU fournies par le turbidimètre. Les expériences d'injection de SusP sont menées avec une concentration d'injection constante $\mathrm{C}_{0}=1 \mathrm{~g} / \mathrm{L}$ et une vitesse d'écoulement de $0,013 \mathrm{~cm} \cdot \mathrm{s}^{-1}$. À la fin de chaque expérience, le matériau (milieu poreux avec particules déposées) remplissant la colonne est soigneusement extrait et divisé en huit sections en fonction des positions des transducteurs (figure 2) afin de mesurer la quantité de particules retenues le long du milieu. La masse de particules déposées dans chaque section est ensuite déterminée selon la procédure décrite par Alem et al. [2]. Pour chaque section, une valeur moyenne de rétention définie comme le rapport entre le volume de particules retenues dans la section et le volume de la section, est calculée permettant de tracer le profil de rétention. 


\section{XVİ̀mes Journées Nationales Génie Côtier - Génie Civil \\ Le Havre 2020}

\section{Evaluation du dépôt de particules par essais de filtration (méthode destructive)}

La figure 3 présente les courbes de restitution et les profils de rétention, expérimentaux et simulés (les différentes équations de la simulation de la restitution ainsi que de la rétention, sont données par ALEM et al. (2013) des SusP injectées. Pour les trois milieux poreux (figure 3a), le taux de particules restituées augmente rapidement pour atteindre un plateau après un volume injecté d'environ deux volumes de pores (stade initial) puis augmente de façon quasi linéaire jusqu'à la fin de l'expérience (stade transitoire). L'évolution de la phase transitoire indique une détérioration progressive de la capacité de filtration du milieu avec le temps. En comparant les courbes des trois milieux, la valeur de la concentration relative au plateau est la plus faible pour Robu (14\%), légèrement plus grande pour Tobermorite $(14,5 \%)$ et très grande pour GB (32\%). Robu et Tobermorite sont les plus filtrants des trois milieux En effet, les particules en suspension accèdent à une partie des sites de rétention de la microporosité, augmentant ainsi leur probabilité de capture, ce qui explique les capacités de filtration plus élevées des deux milieux DPP La saturation rapide des sites de rétention, qui sont moindres dans le milieu SPP que dans les deux milieux DPP, conduit à un colmatage rapide du milieu SPP (la présence de microporosité dans Robu et Tobermorite fournit des sites de rétention supplémentaires retardant le colmatage.
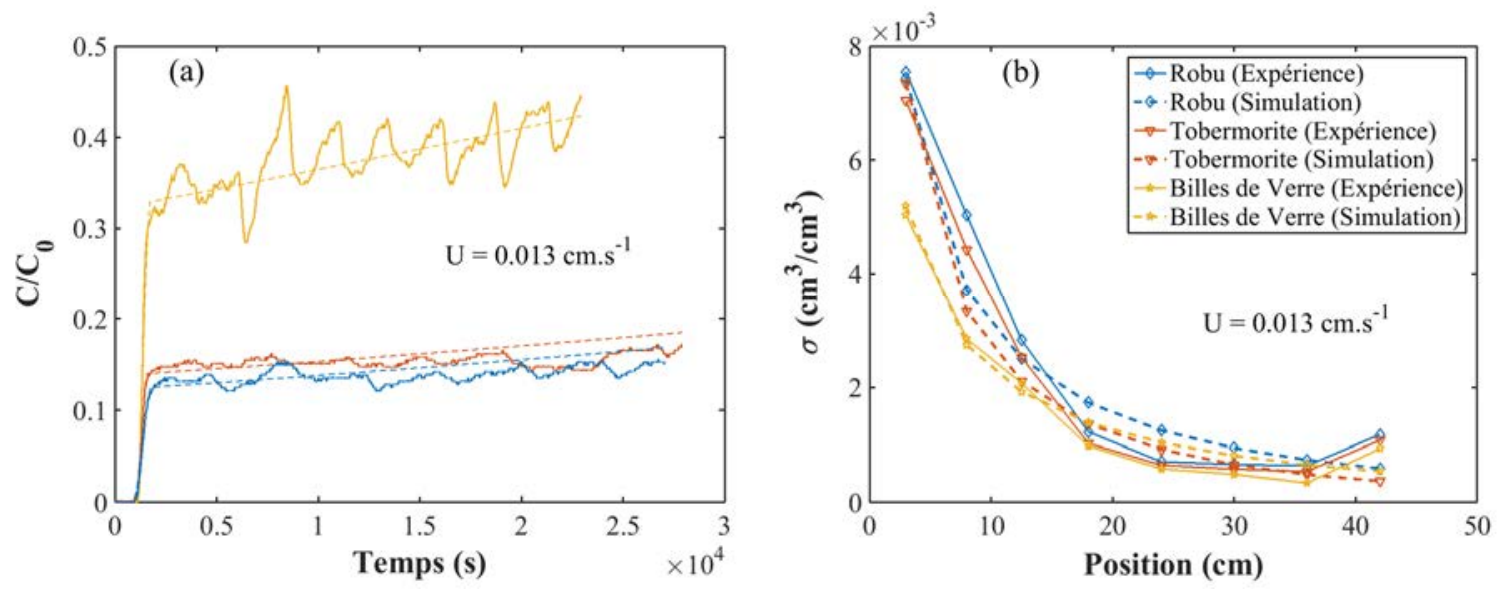

Figure 3. (a) Courbes de restitution et (b) profils de dépôt, expérimentaux et simulés.

La figure $3 \mathrm{~b}$ montre que le dépôt de particules est distribué de manière non uniforme dans l'espace. En effet, le dépôt est plus important dans les premiers centimètres, près de l'entrée du milieu (pos1, voir figure 2), et diminue avec la profondeur. La comparaison des profils de dépôt pour les trois milieux poreux montre que la rétention à une profondeur fixe est globalement la plus élevée pour Robu, légèrement inférieure pour Tobermorite, et est la plus faible pour les BV. Notons que, malgré la porosité totale $(70 \%)$ et la microporosité (31\%) élevées de la Tobermorite, la petite taille d'une grande partie des micropores dans les grains de la Tobermorite suggère un accès moindre des SusP à ces 


\section{Thème 3 - Instrumentation, mesures, imagerie et télédétection}

pores, ce qui réduit la probabilité de leur capture. Cependant, pour le Robu, les tailles des micropores sont suffisamment grandes favorisant l'accès des particules à une grande partie de ces pores, ce qui explique les valeurs élevées du dépôt.

\section{Détection ultrasonore du dépôt de particules}

L'étude de la transmission des ondes acoustiques à travers les trois milieux poreux permet de suivre l'évolution des signaux temporels dans une bande passante déterminée en fonction de la fréquence centrale $\mathrm{du}$ transducteur (figure 4). La translation des transducteurs le long de l'axe horizontal permet d'enregistrer les signaux acoustiques dans huit positions différentes le long de la colonne (voir figure 2). Les matériaux ne sont pas tout à fait disposés de la même manière le long de la colonne (remplissage manuel), donc les signaux initiaux relevés à chaque position sont légèrement différents, ce qui s'avère normal étant donné que le positionnement des grains se fait de façon aléatoire. La figure 4 présente la modification de l'amplitude des signaux temporels (en Volts) pour le Robu (les modifications pour les deux autres matériaux sont similaires avec des valeurs différentes, et ne sont pas présentées ici pour des soucis de simplification) avant et après 2 heures et 7 heures d'injection (pour une meilleure lisibilité seules les première et dernière positions sont montrées, voir figure 2). On constate que l'amplitude du signal diminue après l'injection, pour toutes les positions. Le dépôt de particules dans le milieu provoque une absorption impliquant des pertes d'énergie et donc une diminution d'amplitude. Cependant cette diminution est moins prononcée pour la position 8 (figure 4b), ce qui est dû au fait que les particules se déposent de moins en moins à l'approche de la sortie de la colonne (cf. paragraphe 4).
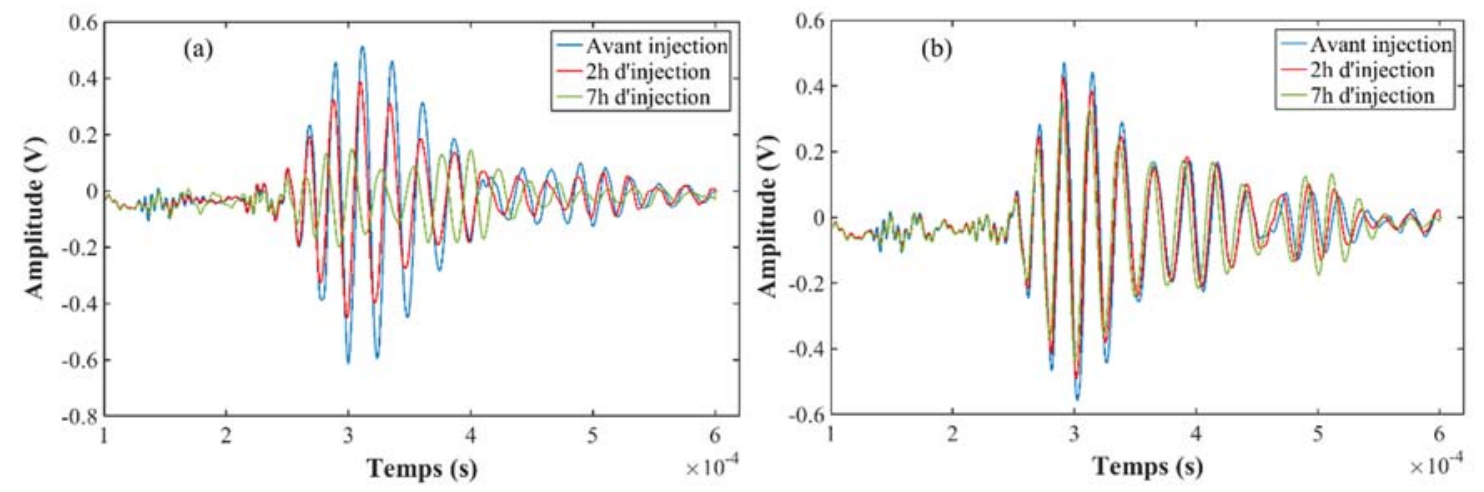

Figure 4. Signaux temporels du Robu avant et durant l'injection de particules (a) pour la position 1, (b) pour la position 8.

\section{Comparaison entre l'évaluation ultrasonore et la méthode destructive}

Les signaux temporels transmis à travers les matériaux étudiés ont été interprétés afin de modéliser le colmatage. Ces signaux, dans ce cas, indiquent l'énergie des ondes transmises à travers les milieux poreux. Une variation des signaux avant et pendant les 


\section{XVI'̀mes Journées Nationales Génie Côtier - Génie Civil \\ Le Havre 2020}

injections de particules est visualisée (figure 4), et elle s'explique par une perte d'énergie transmise à travers le milieu due aux modifications des caractéristiques du milieu à cause du dépôt. Suite à cela, nous introduisons l'énergie du signal qui est fréquemment utilisée dans le traitement du signal, donnée par :

$$
E=\int|x(t)|^{2} d t
$$

avec $x$ (en volts) est le module de la mesure réalisée au carré (dans le cas présent, c'est l'amplitude du signal transmis). Comme indiqué précédemment, les signaux à chaque position le long de l'axe horizontal du modèle, diffèrent légèrement en raison du remplissage manuel du matériau dans la colonne. Pour cette raison, le calcul de l'énergie est effectué en prenant comme référence le signal relevé avant l'injection (milieu propre). L'évaluation du dépôt correspond donc à la différence des énergies avant $(\mathrm{Eb})$ et après ( $\left.\mathrm{E}_{\mathrm{a}}\right)$ chaque heure de l'injection $\Delta \mathrm{E}=\mathrm{E}_{\mathrm{b}}-\mathrm{E}_{\mathrm{a}}$.

Nous représentons sur la figure 5, la variation spatiale de la rétention $\sigma$ et de la variation d'énergie $\Delta \mathrm{E}$ pour les trois matériaux. L'accumulation de particules dans les premiers centimètres du milieu est confirmée par une grande variation du signal transmis après injection. Près de la sortie de la colonne, $\Delta \mathrm{E}$ continue de varier de la même manière que le profil de dépôt pour les trois matériaux, cependant, à partir de la quatrième position environ (à $20 \mathrm{~cm}$ ), la variation devient de plus en plus faible par rapport à l'entrée. Notons que, $\Delta \mathrm{E}$ est moins important dans la Tobermorite malgré un dépôt élevé (le dépôt affecte très peu ses caractéristiques acoustiques).

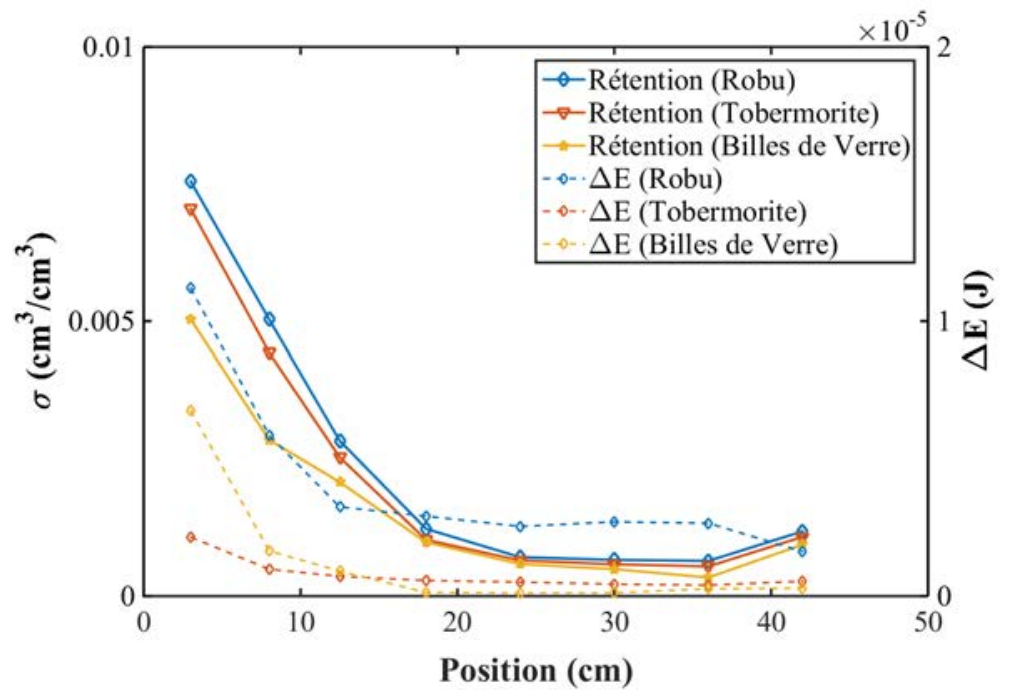

Figure 5. Comparaison entre les profils de dépôt et les profils d'énergie le long de l'axe horizontal $x$ pour les trois matériaux.

\section{Conclusions}

Le phénomène du colmatage dans les milieux poreux est assez compliqué par les différents processus qui le décrivent. Le dépôt affecte principalement la porosité et la 


\section{Thème 3 - Instrumentation, mesures, imagerie et télédétection}

perméabilité, qui sont les paramètres les plus importants dans les milieux poreux. Plusieurs paramètres peuvent être impliqués dans le processus du dépôt de particules dans un milieu poreux saturé. La comparaison de trois matériaux différents (deux à double porosité et le troisième à simple porosité) a permis de déduire la grande importance de la structure du matériau dans le dépôt. La capacité de rétention du milieu dépend fortement de la tortuosité, et de la taille (longueur et diamètre) des micropores (pour la Tobermorite, malgré une porosité très importante, sa capacité de rétention est moins importante que le Robu qui contient des micropores plus gros et plus profonds).

Concernant le colmatage, un bon accord est observé entre l'évaluation destructive et la détection ultrasonore non destructive basée sur la transmission des ondes à travers le milieu. La variation des propriétés acoustiques (signaux temporels et énergie du signal transmis) avec le dépôt a été présentée. En effet, les courbes de variation d'énergie suivent la même allure que les profils de dépôt. De ces résultats, on déduit la sensibilité des méthodes acoustiques au phénomène de colmatage.

\section{Références bibliographiques}

ALEM A., ELKAWAFI A., AHFIR N., WANG H. (2013). Filtration of kaolinite particles in a saturated porous medium: hydrodynamic effects, Hydrogeology Journal; Vol. 21, pp 573-586. https://doi.org/10.1007/s10040-012-0948-x

BAI R., ALEM A., FRANKLIN H., TINEL A., WANG H., KACHKOUCH F., TAIBI S., BIZET L. (2018). Ultrasonic characterization of particle retention by a double porosity medium, Environmental Technology, Vol. 40(9), pp 1166-1177. https://doi.org/10.1080/09593330.2017.1417494

BERRYMAN J.G., WANG H.F. (2000). Elastic wave propagation and attenuation in a double-porosity dual-permeability medium, Int. J. Rock Mech. Min. Sciences, Vol. 37, pp 63-78. https://doi.org/10.1016/S1365-1609(99)00092-1

BIOT M.A. (1956). Theory of propagation of elastic waves in a fluid-saturated porous solid. I. Low-frequency range, J. Acoust. Soc. Am., Vol. 28, pp 168-178. https://doi.org/10.1121/1.1908239

KIMURA M. (2006). Frame bulk modulus of porous granular marine sediments, The Journal of the Acoustical Society of America, Vol. 120, 699. https://doi.org/10.1121/1.2211427 MOGHADASI J., MÜLLER-STEINHAGEN H., JAMIALAHMADI M., SHARIF A. (2004). Model study on the kinetics of oil field formation damage due to salt precipitation from injection, J. Pet. Sci. Engineering, Vol. 43; pp 201-217. https://doi.org/10.1016/j.petrol.2004.02.014

WARREN J.E., ROOT P.J. (1963). The behavior of naturally fractured reservoirs, J. Soc. Pet. Eng., Vol. 3, pp 245-255. https://doi.org/10.2118/426-PA

WILSON R.K, AIFANTIS E.C. (1984). A double porosity model for acoustic wave propagation in fractured porous rock, Int. J. Eng. Sciences, Vol. 22, pp 1209-1217. https://doi.org/10.1016/0020-7225(84)90124-1 